\title{
Stage reduced counter-rotating axial compressor for jet engine
}

\author{
F. Ferdaus ${ }^{1, *}$, Nitish Kumar ${ }^{2}$, G. Sakthivel ${ }^{1}$, and N. Raghukiran ${ }^{1}$ \\ ${ }^{1}$ Centre for Automation, School of Mechanical Engineering, Vellore Institute of Technology, Chennai, India \\ ${ }^{2}$ Kings Cornerstone International College, Chennai, India
}

Received: 25 January 2021 / Accepted: 30 March 2021

\begin{abstract}
Variation in the states of system, mass flow and pressure are some of the disturbances which are experienced by the compressors in the jet engine under working condition. One of the main factors that influence the efficiency of a jet engine is the pressure ratio. In order to achieve the required pressure ratio, we should have relatively a greater number of stages in the compressor that leads to an increase in the weight of the engine. The stator and rotor are the essential parts of an aircraft's axial compressor. CFD is used in order to evaluate the pressure ratio. In this paper, we are going to analyze a three-stage compressor instead of an actual six-stage compressor. The mass flow rate inside the control system can be used to maintain the stability of the system. Compressor weight and pressure ratio at each stage can be reduced if we have a clockwise and anti-clockwise rotating rotor. With the use of a universal gear system, the two clockwise rotors and one anti-clockwise rotor were analyzed. The main outlook of this work is to show the maximum pressure ratio of the compressor at the outlet with our desired configurations. In conclusion, it was shown that the weight of the aircraft engine can be effectively reduced.
\end{abstract}

Keywords: Axial flow compressor / Propulsion / Gas turbine engine / Computation fluid dynamics / CAD / Pressure ratio / CFD

\section{Introduction}

There are many functions for the compressor section of a gas turbine engine. The primary function of this region is to supply pressurized air to the combustion chambers [1]. The air entering the compressor from the air inlet duct must be pressurized and then transferred to the burners at the required quantity and pressures. The secondary function of the compressor is to supply the bleed air. From various pressure stages, the bleed air is taken. Before the air entering the combustion chamber, the pressure of the air should be increased in order to achieve greater efficiency that is where the work of a compressor arrives. Regarding the operation of the compressor, it is rotated by a shaft which is powered by the turbine of the aircraft engine. Due to the rotation of the stages in the compressor section, the pressure and the temperature of the air are increased stage by stage [1].

The centrifugal type of compressor is the first one to be used in jet engines and they are still in use in turbojet and turboshaft engines. The airflow is diverted perpendicular to the axis of rotation by using the centrifugal compressor [2]. The air is collected in the scroll/volute once after it has been moved by the rotating impeller. Between the impeller

\footnotetext{
* e-mail: Fadyfithu@gmail.com
}

and the volute, there may be a diffuser. A small pipe is an inlet to a centrifugal compressor $[2,3]$. The valve, stationary vanes, or airfoils are some of the features included in the inlet area. The pressure and temperature instrumentation are also housedin the inlet area. When it comes to the control of the centrifugal compressor, the aforementioned features play an important role. The centrifugal impeller is the principal component that makes the compressor centrifugal [2,3]. The energy of the working gas is raised gradually by the impeller which consists of a rotating set of vanes. This is similar to that of the axial compressor but in this process, due to the increasing radius of the impeller, we can reach higher energy levels and velocities. In a modern high-end centrifugal compressor, the exiting gas from the impeller cruises nearly at the speed of sound. On the contrary, the common and most available configurations of impellers are the open- visible blades, shrouded- with splitters.

Generally, adjustable guide vanes or rotating guide vanes are used for capacity control in a centrifugal compressor [3]. The rotational guide vanes are always placed at the eye of the impeller. For the inception of the surge in a centrifugal compressor, the main and key component is the radical diffuser vanes $[3,4]$. These radial diffuser vanes operate at high rotational speeds, being the main reason for the inception of a surge in the compressor. In 1975 , the radial vaned diffuser has been attributed to the 
fact of rotating surge and stall. An impeller, a compressor manifold, and a diffuser are the main components comprised by a centrifugal-flow compressor. Relatively high-pressure (8:1) rise per each stage can be seen in a typical centrifugal compressor. Due to the efficiency concerns, the centrifugal compressors are limited to two stages [4]. So, the airflow leaving the passing through these stages will have high pressure. As previously discussed, while the airflow passes through each of the stages in the compressor, the pressure is increased by a ratio of about $8: 1$. All the aforementioned points are operating due to the centrifugal action. Though, the centrifugal compressor has some advantages, in modern-age, this type of compressor is not used as a primary compressor in the aircraft.

Airflow is diverted parallel to the axis of rotation in the axial compressor [5]. A series of airfoils consisting of rotors and stators are used in order to provide more efficiency. The rotors rotate at high speeds and are connected to the central shaft. Stators are fixed to the outer casing and help to increase the pressure [5]. One rotor added toone stator gives the equivalent resultas one stage of the compressor. The stators are stationary parts and the rotors are rotating parts of the compressor. The function of the stator is to guide the airflow to the next stage rotor in order to obtain maximum efficiency. The function of the rotor is to increase the pressure of the gas by cutting through the airflow [6]. In this case, the working fluid used in the compressor is air. The pressure is increased stage-by-stage resulting in rise inthe temperature of the gas. Due to the rotation of the rotors in each stage, the flow of air is cut in order to increase both of these factors. The governing factors for this process are the rotation of the shaft which is achieved by the turbine, design of the stator and rotor, and the number of stages in order to obtain the optimum efficiency [7]. In order to deliver the air to the inlet of the axial compressor, we use inlet guide vanes. The potential for unwanted emissions can be minimized by using this inlet guide vanes as it helps to maintain proper fuel to air ratio [8]. Variable stator vanes are present between the adjacent rotor blades. The variable stator vanes can be rotated about an axis to direct the airflow towards the compressor [4,8]. The vanes also help in the optimized performance of the compressor by monitoring the quantity of airflow in the compressor.

The counter-rotating compressor is a design that is yet to be deployed in commercial aircraft [9]. Hindustan Aerospace Limited analyzed the advantages and disadvantages of the counter-rotating compressor. In the present work, finite element modeling of the counter-rotating compressor was analyzed in order to bring out the total pressure and velocity distribution over the design of the compressor. There will be a change only in the blade profile and the rotation of the blades when it is compared to the actual design. An attempt has been made to come up with a compact and light-weight alternative to the existing axial compressor by optimizing its design. One of the major problems that are faced by exiting axial flow compressor is its geometric complexity which tends to reduce the performance of the engine $[10,11]$. Secondly, we need to increase the number of stages in the axial flow compressor to get a high-pressure ratio which in turn increases the weight of the aircraft resulting in the consumption of more amount of fuel [10]. Finally, the third problem is that the airflow is neutralized by the stator blades and that requires a high amount of power to start the axial flow compressor.

Traditionally, centrifugal compressors were used for the compressor region of the engine. In the later times, the centrifugal compressors were replaced with the axial compressorsthat arecharacterized by a lot of stages that canresult in a relatively higher weight of the overall system. In this particular work, an attempt has been made to analyze anaxial counter-rotatingcompressor that could potentially overcome the prevailing problems posed by the use of axial compressors [10,11].

The compressor provides the pressurized air to the turbine and it is located in the front section of the engine $[9,10]$. When we use a conventional axial stage compressor, the pressure of the air increases substantially from one stage to the other. The rotor blades in the compressor help in converting the mechanical energy to gaseous energy. The main role of the compressor is to produce sufficient energy during the combustion process [9]. Sufficient energy can't be achieved ifthe fuelis burnt in atmospheric conditions. Hence, axial compressor, centrifugal compressor, or axialcentrifugal compressor are the three main types of compressorsused to provide the air at required pressure for effective combustion [11].

Reliable operation of the fan and the turbine blades can be achieved only through the selection of appropriate materials during the manufacturing process. The composite materials were reported to perform better over titanium-based alloys due to their excellent strength and stiffness-to-weight ratio [12]. Three-dimensional weaving of the yarns in the form of fiber-reinforcement can be used to manufacture the airfoil structure of the fan blade [13].

Due to the vibrations, dynamic forces are reported to form which in turn lead to the fatigue of the rotating components and suchphenomenon is referred to as the high cycle fatigue. In one of the reports, during the experiment, the blades were placed at transverse vibration, and resonance condition was used in order to process the crack propagation [14]. The growth of crack during the fatigue test was monitored. In order to determine the stress state, a non-linear finite element method was used. The crack occurring point was the maximum principal stress zone [14]. Above the blade locking piece at a distance of 2-10 mm, cracks were formed at the convex surface of the blade profile during the visual test. For the compressor blades, the transverse vibrations are very harmful because during the blade resonance high stress occurs [14]. The transverse vibrations can be very dangerous for compressor blades due to the associated higher stress levels during the blade resonance.

Wearing that occurs between two surfaces which tend to move in oscillatory motion even of the small amplitudeis known as fretting [15]. The criticalcomponents in fields such as aerospace industry and nuclear-energy establishments, fretting fatigue is an important aspect of the overall safety of the systems. The fretting fatigue occurs at the dovetail roots of the compressor blades and their failure will have a serious impact on the engine integrity $[16,17]$. In a compressor blade, the blade or disk attachment which is also known as the dovetail joint is the most common failure 
which occurs due to the fretting fatigue and that is because of the vibrational loads and the high frequencies which in turn causes the high cycle fatigue condition on that blade resulting in the fretting fatigue. This is most common in the compressor blades made of Ti6Al4V alloydue to the short oscillations and high contact stresses at the interface between the blade and the disk which causes the fretting wear resulting in component failure [18].

The main idea of the counter-rotating compressor is to achieve more pressure by using fewer stages. In such compressors, the weight of each stage of the compressor varies from one another. The counter-rotating compressor eventually reduces the weight of the engine which in turn consumes less fuel. Efficient conversion of pressure can be obtained by using this model of a compressor as one stage rotates clockwise, and the subsequent stage rotates anticlockwise. Efficient balance can be obtained if such type of compressor can be used so that the engine size and design can be made compact. Further, any changes in the atmospheric conditions does not affect the engine performance.

The present work involves a detailed account of the new technique that could be provided to the axial flow compressor of an aircraft engine. The implementation of such technique to the axial compressor is to maintain the pressure ratio in the compressor section of a gas turbine engine that can be housed in a commercial aircraft. The main theoretical background in the implementation of the counter-flow axial compressor is to reduce the compressor stages in the compressor section of the aircraft's engine compartment, increase the pressure output from the compressor, and reducethe total weight of the enginewhich in turn reduces the fuel consumption. The achievable objects with this approach include the maintainability of the pressure ratio in the compressor region which produces the desired pressure ratio by using a lesser number of compressor stages when compared to the other types of compressors. Due to the usage of a compressor with lesser number of stages, the compressor section weight is reduced, which in turn reduces the total weight of the engine consuming a lesser amount of fuel in order to produce the desired thrust.

\section{Methodology}

The principle of any heat engine is to convert heat into work, preferably as efficiently as possible. The ideal gas turbine cycle consists of the following processes and the numerical notation of which is used throughout this chapter are:

This $\mathrm{P}-\mathrm{V}$ graph states that the number line 1-2 represents the frictionless adiabatic compression. The number line 2-3 represents the frictionless constant pressure heating. The number line 3-4 represents the frictionless adiabatic expansion. Finally, the number line $4-1$ represents the frictionless constant pressure heat rejection.

Such cycle can be represented on a pressure-volume diagram, where the area enclosed represents the network output. A typical jet engine runs on Brayton cycle. In the present work, a single spool turbojet engine of $5 \mathrm{KN}$ thrust class was selected with a fixed turbine inlet temperature of $1200 \mathrm{~K}$ from a material and life point of view. For optimum specific fuel consumption, iterations were carried out and

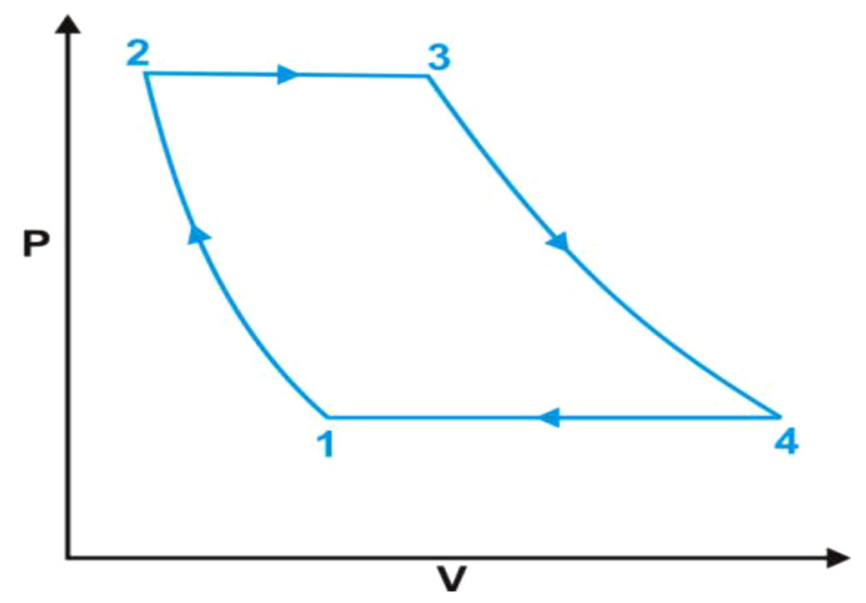

Fig. 1. Gas Turbine $\mathrm{P}-\mathrm{V}$ cycle graph.

air mass flow rate $\left(\mathrm{M}_{\mathrm{a}}\right)$ of $7.75 \mathrm{~kg} \backslash \mathrm{s}$, and compressor pressure ratio (r) of 9 have been adopted. The specific parameters and the values which have been used are presented in Figure 1.

From the compressor pressure ratio, the value of compressor outlet pressure can be calculated as follows:

$$
\begin{gathered}
\mathrm{P}_{02}=\mathrm{r}^{*} \mathrm{P}_{01} \\
\mathrm{P}_{02}=9^{*} 101.325 \\
\mathrm{P}_{02}=911.925 \mathrm{KPa}
\end{gathered}
$$

The compressor outlet temperature was calculated from the relation of temperature and pressure ratio for an isentropic flow,

$$
\begin{gathered}
\mathrm{T}_{02} / \mathrm{T}_{01}=(\mathrm{r})^{(\mathrm{r}-1 / \mathrm{r})} \\
\mathrm{T}_{02} / \mathrm{T}_{01}=(9)^{(0.4 / 1.4)}
\end{gathered}
$$

For air isentropic flow the gamma $=1.4$

$$
\begin{aligned}
& \mathrm{T}_{02}=539.29 \mathrm{~K} \\
& \mathrm{~T}_{03}=1200 \mathrm{~K}
\end{aligned}
$$

For the ideal cycle, a constant pressure combustion process will take place,

$$
\begin{gathered}
\mathrm{P}_{03}=\mathrm{P}_{02} \\
\mathrm{P}_{03}=911.925 \mathrm{~K} \mathrm{~Pa}
\end{gathered}
$$

The mass flow rate of fuel $\left(\mathrm{M}_{\mathrm{f}}\right)$ is calculated from the combustion chamber efficiency relation and substituting the values of mass flow rate of air $\left(\mathrm{M}_{\mathrm{a}}\right)$, Turbine inlet temperature $\left(\mathrm{T}_{03}\right)$, calorific value $\left(\mathrm{C}_{\mathrm{V}}\right)$, and compressor outlet temperature $\left(\mathrm{T}_{02}\right)$.

From the heat balance equation across combustor,

$$
\begin{aligned}
& \text { Compressor's exit heat energy } \mathrm{Q}_{\mathrm{c}}=\mathrm{M}_{\mathrm{a}} * \mathrm{C}_{\mathrm{p}} *\left(\mathrm{~T}_{02}\right) \\
& \text { Combustion chamber exit heat energy } \mathrm{Q}_{\mathrm{b}} \\
& =\left(\mathrm{M}_{\mathrm{a}}+\mathrm{M}_{\mathrm{f}}\right) * \mathrm{C}_{\mathrm{pg}} *\left(\mathrm{~T}_{03}\right)
\end{aligned}
$$




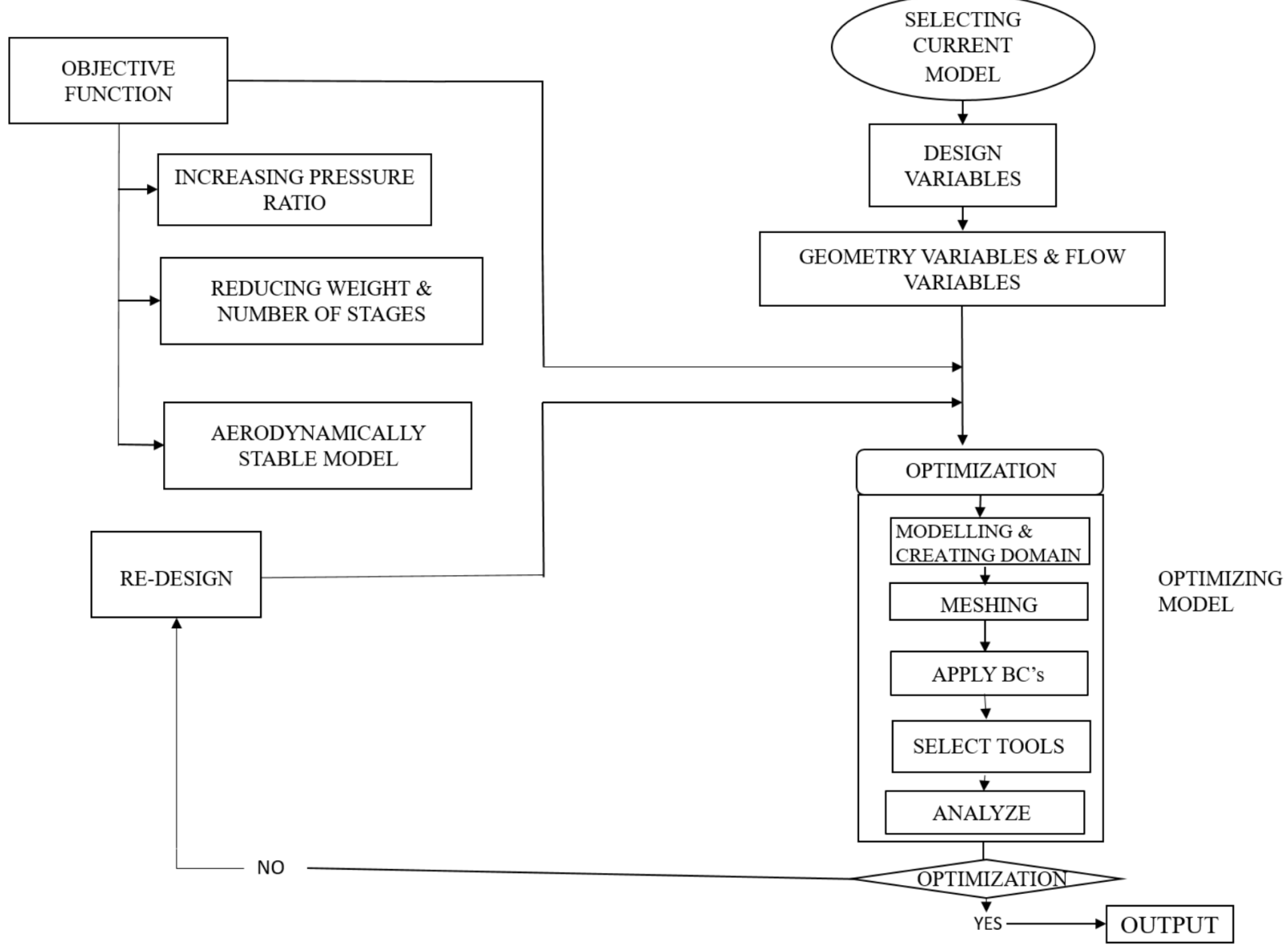

Fig. 2. Flowchart of the depiction of systematic development for the axial compressor.

Amount of heat added in combustion chamber $=\mathrm{Q}_{\mathrm{b}}-\mathrm{Q}_{\mathrm{c}}$ $=\mathrm{M}_{\mathrm{f}} * \mathrm{C}_{\mathrm{V}} * \eta_{\mathrm{b}}$

$$
\mathrm{M}_{\mathrm{f}}=0.125 \mathrm{~kg} / \mathrm{s}
$$

Nozzle inlet temperature $\left(\mathrm{T}_{04}\right)$ is calculated from the mechanical efficiency formula by substituting the values of turbine inlet temperature $\left(\mathrm{T}_{03}\right)$, the mass flow rate of fuel $\left(\mathrm{M}_{\mathrm{f}}\right)$ and air (Ma), mechanical efficiency $\left(\eta_{\mathrm{m}}\right)$, and compressor outlet temperature $\left(\mathrm{T}_{02}\right)[19,20]$.

From the spool matching equation or turbine and compressor matching equation,

$$
\begin{gathered}
\mathrm{C}_{\mathrm{pg}} * \eta_{\mu} *\left(\mathrm{M}_{\mathrm{a}}+\mathrm{M}_{\mathrm{f}}\right)\left(\mathrm{T}_{03}-\mathrm{T}_{04}\right)=\mathrm{C}_{\mathrm{pa}}\left(\mathrm{T}_{02}-\mathrm{T}_{01}\right) \mathrm{Ma} \\
\mathrm{T}_{04}=1200-((1.05 *(539.29-288.15) * 7.75) / \\
(1.148 * 1 *(7.75+0.125))) \\
\mathrm{T}_{04}=973.286 \mathrm{~K}
\end{gathered}
$$

The turbine exit pressure $\left(\mathrm{P}_{04}\right)$ was obtained from the turbine efficiency formula by substituting the values of turbine inlet temperature $\left(\mathrm{T}_{03}\right)$, the efficiency of the turbine $\left(\eta_{t}\right)$, turbine exit pressure $\left(\mathrm{P}_{04}\right)$, and turbine inlet pressure $\left(\mathrm{P}_{03}\right)$.

$$
\begin{gathered}
\mathrm{T}_{03}-\mathrm{T}_{04}=\mathrm{T}_{03} \eta_{t}\left(1-1 /\left(\mathrm{P}_{03} / \mathrm{P}_{04}\right)^{(0.33 / 1.33)}\right) \\
\mathrm{P}_{04}=1-(1200-973.286) /(1200 * 1)^{(1.33 / 0.33) * 911.925} \\
\mathrm{P}_{04}=392.38 \mathrm{Kpa}
\end{gathered}
$$

As shown in the flowchart (Fig. 2), the first step would be selecting the existing model. Secondly, the design variables are identified. Design variables are entities that can change the shape of the model within a specific range during optimization. The next step would be the geometry and flow variables which are similar to the design variables. The fourth step would be the optimization process where the designing and analysis was conducted. The result wasthen projected as an optimized model. On the other hand, if the optimization does not converge, theniteration of the entire process (redesigning) was done Table 1.

\subsection{Axial compressor design for gas turbine}

The vital dimensions for the existing standard axial compressor and the proposed design are given in Table 2 . 
Table 1. Basic parameters and the values used for the current study.

\begin{tabular}{lll}
\hline S. No & Parameters & Value \\
\hline 1 & Inlet pressure $\mathrm{P}_{01}$ & $101.325 \mathrm{~K}$ Pascal \\
2 & Inlet temperature $\mathrm{T}_{01}$ & $288.15 \mathrm{~K}$ \\
3 & Inlet density $\rho_{01}$ & $1.225 \mathrm{~kg} \backslash \mathrm{m}^{3}$ \\
4 & Turbine inlet temperature $\mathrm{T}_{03}$ & $1200 \mathrm{~K}$ \\
5 & Inlet mass flow rate $\mathrm{M}_{\mathrm{a}}$ & $7.75 \mathrm{~kg} \backslash \mathrm{sec}$ \\
\hline
\end{tabular}

Table 2. Comparison of the design parameters of existing and the proposed design.

\begin{tabular}{lll}
\hline & Existing design & Proposed design \\
\hline Height of the blade at the first stage & 58 & 58 \\
The radius of the blade & 85 & 85 \\
Profile of the blade & $\mathrm{R} 18, \mathrm{R} 15, \mathrm{R} 21$ & $\mathrm{R} 21, \mathrm{R} 13$ \\
Width of the blade & 28 & 25 \\
Height of the compressor hub without the blades & 130 & 130 \\
The dimension of the stator and rotor & Stator: $54,42,31,13$ & $50,43,37,28,14,23$ \\
& Rotor: $44,33123,12$ & \\
\hline
\end{tabular}

The values presented in Table 2 gives a direct comparison of existing and the proposed designs. Some of the important components from the design aspect, such as the height of the blade, the radius of the blade, profile of the blade, breath of the blade, and height of the compressor hub without the blade along with the dimensions of the stator and rotor were compared.

The Dassault Systèmes CATIA V5 software package was employed for planning the design of the compressor, owing to its dependability and user-friendliness. The part design involved the general framework in relevance to several 3D and 2D tools like line, profile line, constraints pad, and revolvefeatures in CATIA. The given Figure 3a, b, c, d, e, f represents the existing model of the axial compressor such as multi-view of the axial compressor, section view of existing and counterclockwise, closed and color variation with exiting axial compressor, and proposed design of axial compressor.

\section{Results \& discussion}

Consequently, the boundary condition of the existing models and proposedcounter-rotated compressor are presented in Table 3. The data in Table 3 represents the comparison of the boundary conditions for the existing compressor model and the proposed model. With this comparison, the fluid medium, reference pressure, reference temperature, inlet total pressure, outlet static pressure, wall, clockwise rotating compressor speed, and anticlockwise rotating compressor speed of the existing and new models are compared.

\section{The boundary conditions for the proposed model: counter-rotating compressor}

Subsequent tothe application of boundary conditions, the analysis of both the compressor models was followed by evaluating the results of static pressure, total pressure, and velocity distribution across the structure (Fig. 2).

\subsection{Analysis of existing axial compressor model}

The static pressure experienced by the three stages of the axial compressor is shownin Figure 4. The first stage consists of one rotor and a stator and the rotor rotates in the clockwise direction. This clockwise rotating rotor consists of 30 blades and the stator associated with it consists of 33 blades. Particularly, the prevailing pressure helps in playing a major role which induces deformation and the development of the stresses. In other words, this is also called a static structural model. The pressure has increased at each stage in this image.

Secondly, Figures 4 and 5 show the contour plots in the middle portion of the compressor and they indicate the static pressure region associated with that part. Fromthe aforementioned diagram, it can be observed that the pressure increases from one stage to the other of the rotor. In Figures 4 and 5, a close look at the midplane, the total pressure distribution from the aforementioned diagram can be observed. There is a relative pressure rise across each stage for up to 7950-3600 Pa.

In Figure 6, the depiction of the distribution of velocity across the compressor is shown and it is evident that the velocity also increases from one stage to another. The 

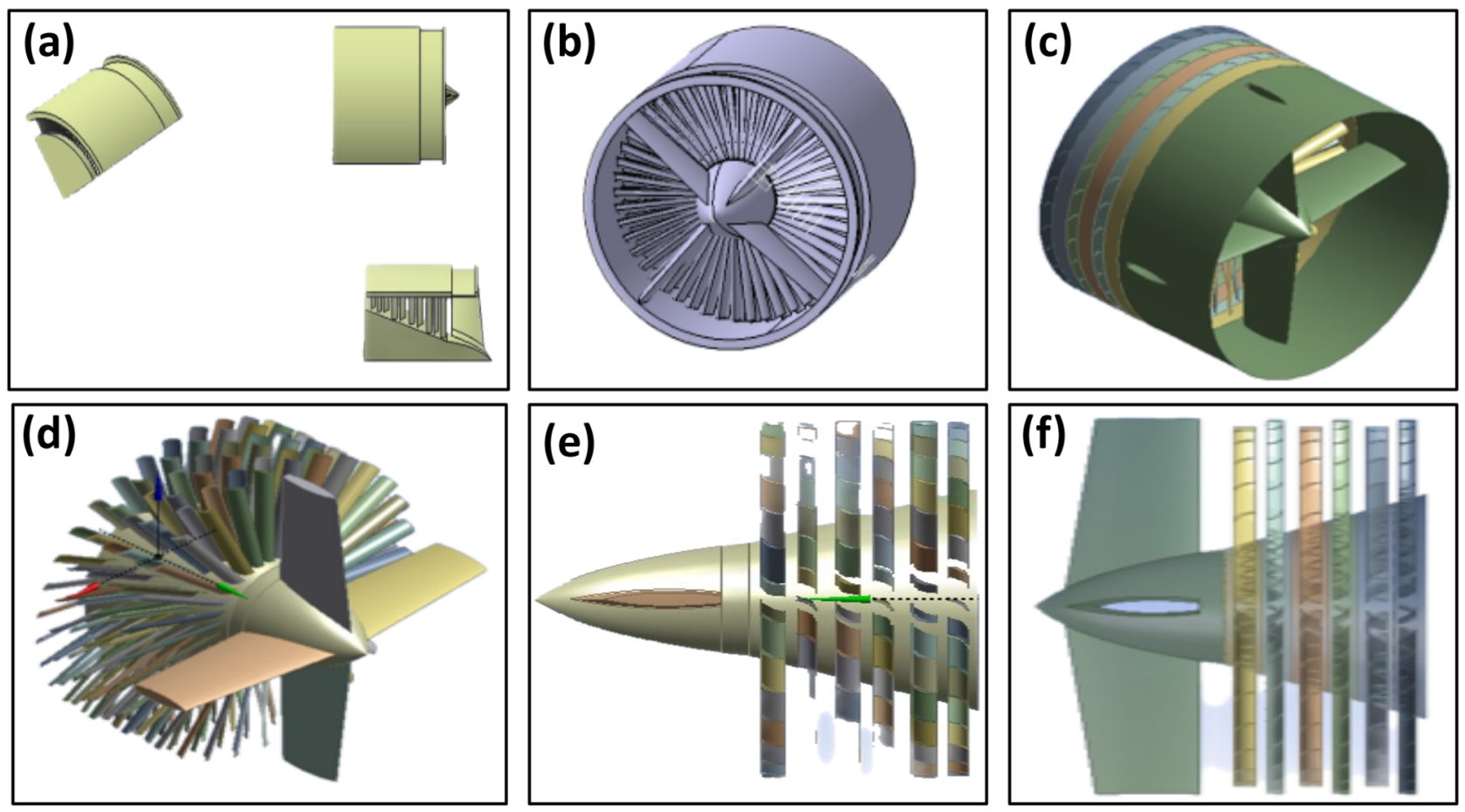

Fig. 3. (a) Multi-view of the existing model, (b) Clockwise axial compressor, (c) axial compressor model with color variation, (d) section-view of the existing axial compressor, (e) counter-rotating compressor, and (f) section-view of the counter-rotating compressor.

Table 3. The boundary conditions for the existing compressor model and the proposed model.

\begin{tabular}{lll}
\hline Fluid medium & Air ideal gas & Air ideal gas \\
\hline Reference pressure & 1.013 bar & 1.013 bar \\
Reference temperature & $300 \mathrm{~K}$ & $300 \mathrm{~K}$ \\
Inlet total pressure & $0 \mathrm{~Pa}$ (assumed) & $0 \mathrm{~Pa}$ (assumed) \\
Outlet static pressure & $0 \mathrm{~Pa}$ & $0 \mathrm{~Pa}$ \\
Wall & $\mathrm{No} \mathrm{Slip} \mathrm{conditions}$ & No Slip conditions \\
Clockwise rotating compressor speed $(\mathrm{rpm})$ & 7000 & 7000 \\
Anti-clockwise rotating compressor speed $(\mathrm{rpm})$ & - & 7000 \\
\hline
\end{tabular}

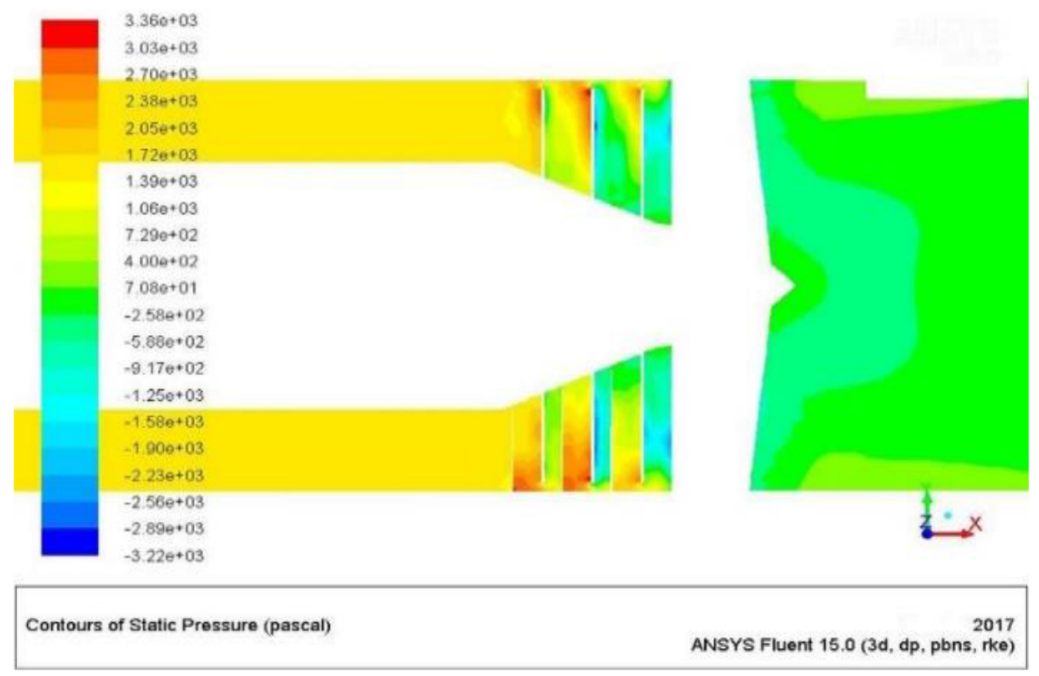

Fig. 4. Contours of static pressure (Units: Pa). 


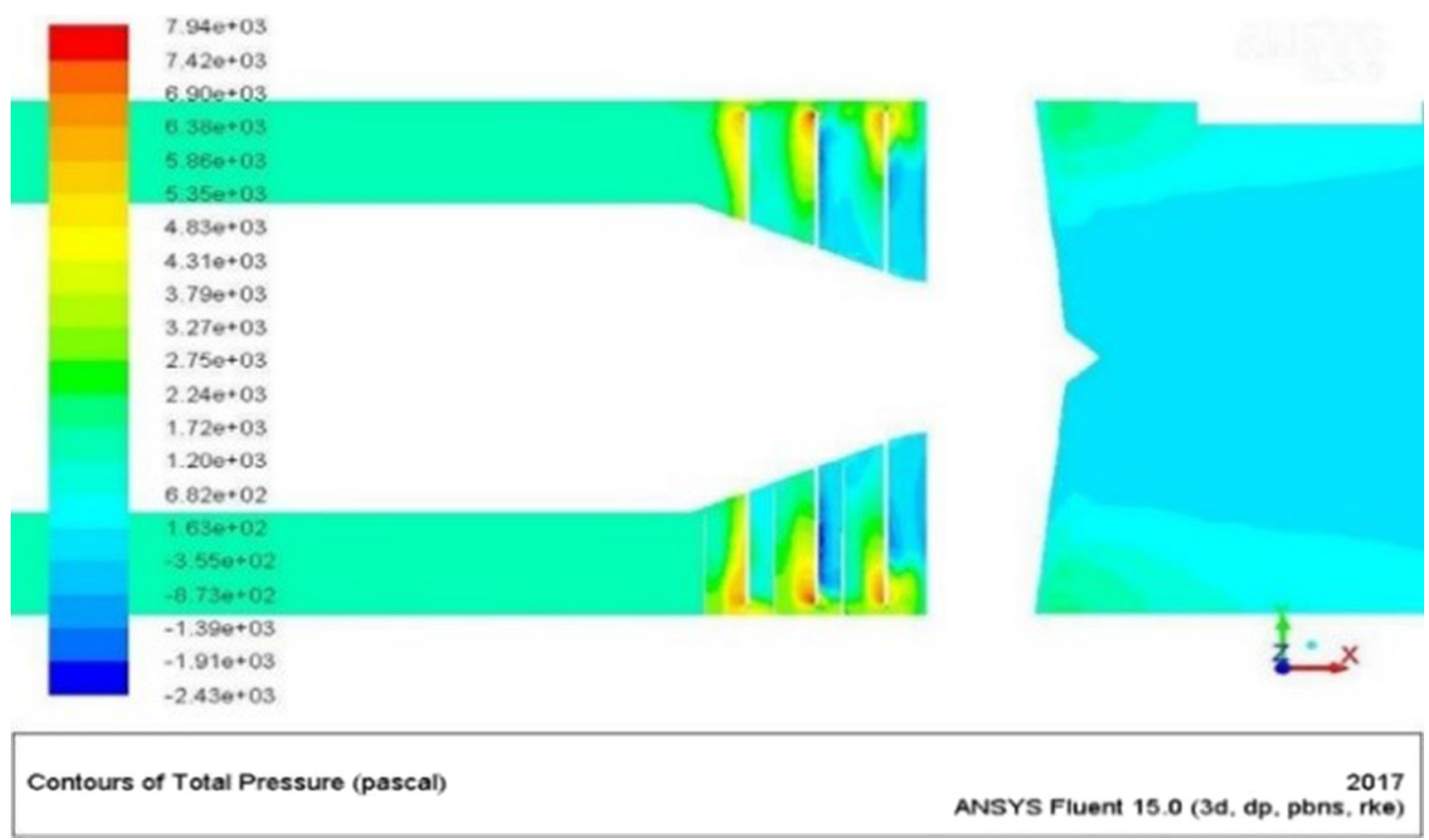

Fig. 5. Contours of total pressure (Units: Pa) in the existing model.
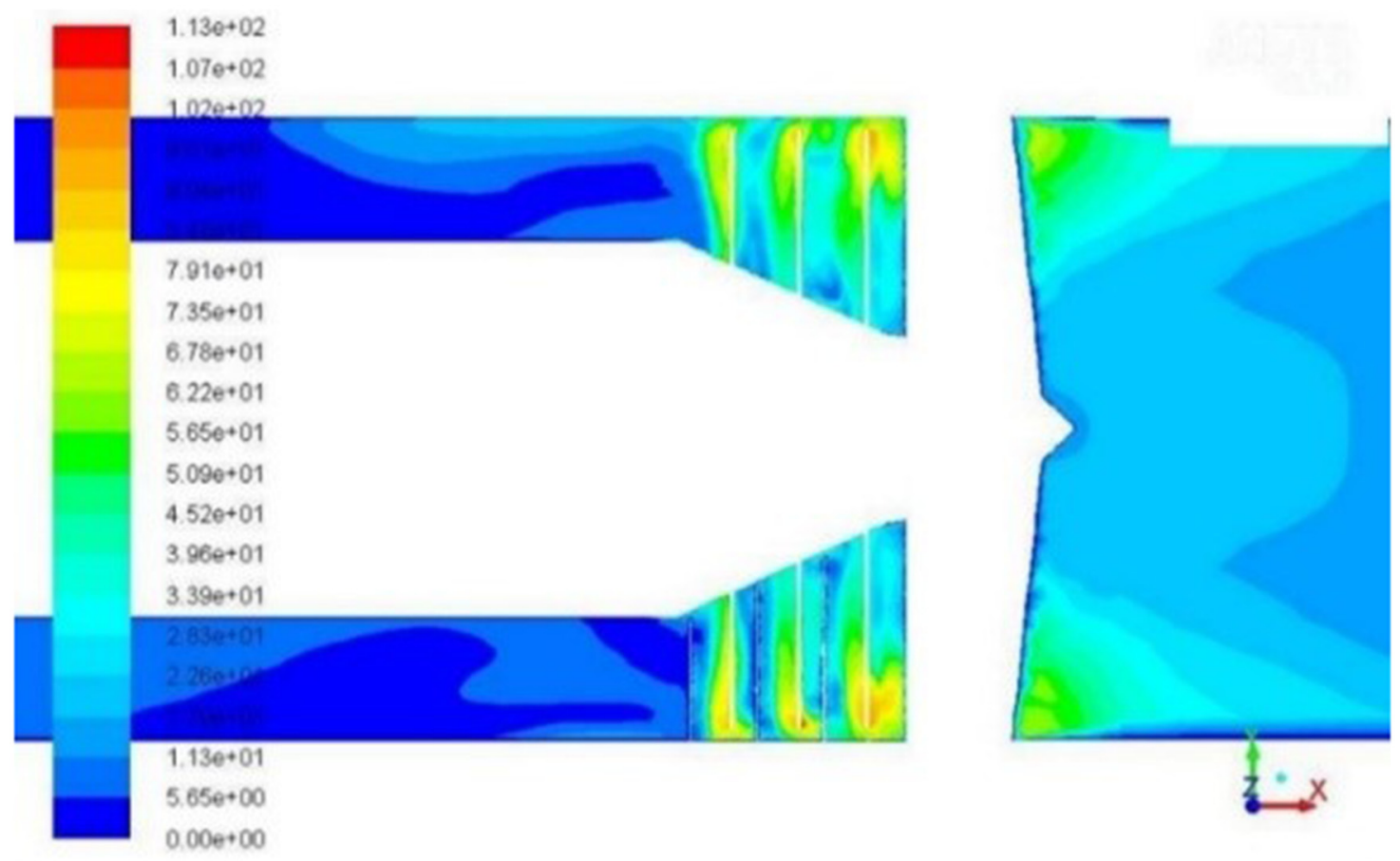

Fig. 6. Velocity magnitude contours of the existing model. 
simulated velocity contours also represent, at the midplane, it can be observed that the total velocity distribution from the aforementioned diagram.

Finally, by the means of velocity magnitude, the velocity vectors of the compressor can be observed as shown in Figure 7. In precise, the velocity vector tell about the rate of change of position of an object and the magnitude of the velocity vector tells the speed of the object.

\subsection{Analysis of axial counter rotating compressor}

This type of compressor also consists of three stages and each stage will be provided with 1 clockwise and an anticlockwise rotor. The clockwise rotor will consist of 30 blades and the anti-clockwise rotor will consist of 33 blades for its function. Static pressure and the velocity of the corresponding air is increased by the clockwise rotor. To achieve an increase in the total pressure, this clockwise

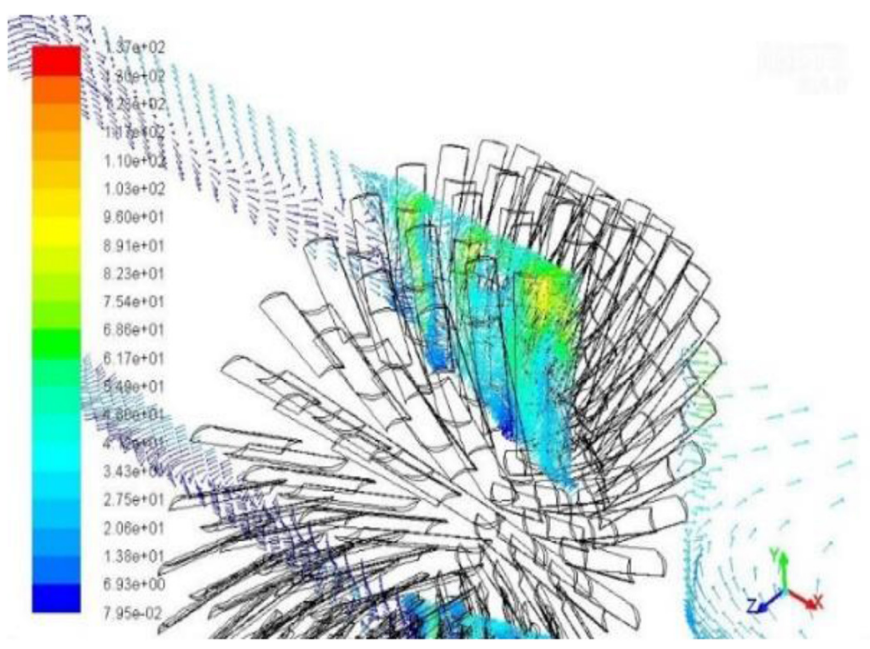

Fig. 7. Velocity vectors colored by velocity magnitude for the existing model. stage air is later passed through the anti-clockwise rotor wherein the velocity is decreased, and the pressure is increased.

The Figure 8 portrays the total pressure distribution across the compressor. This can be used as evidence to mention that the pressure increases across each stage of the rotor. The relative increase of pressure from one stage to another would be of 9150-2800 Pa.

Figure 9 is used to portray the static pressure difference throughout the counter-rotating compressor. Further, it is also a proof that the pressure increases across each stage of the rotor. The pressure plays a major role which supports deformation and helps in creating stress in the entire model and simulation till it reaches the equilibrium state.

Figure 10 is used to brief out the velocity distribution across the compressor. Further, from the image, it can be concluded that the velocity also increases from one stage to the other.

However, this simulation image (Fig. 11) represents the velocity magnitude, and it can be observed that the velocity vectors of the compressor in the above-mentioned diagram. Mostly the velocity vector tells about the rate of change of position of an object and the magnitude of the velocity vector tells the speed of the object.

\subsection{Result comparison}

The Figure 12 compares the results of pressure difference in the existing and counter-rotating compressor. It shows that the pressure have been increased at each stage of the modified model compared to the existing axial compressor.

The regular axial compressor pressure ratio at 3 stage is 7.95. (2.6 per stage) and the counter rotating rotor pressure ratio at 3 stage is 9.15 . (3.1 per stage) as shown in the above figure number 12. From the above comparison, it is clear that having two-stage contra-rotating rotor it is almost equivalent to three stage regular axial compressor. The shape and size of the engine will be compact, so that it can have an effective streamlining and balancing of the aircraft.

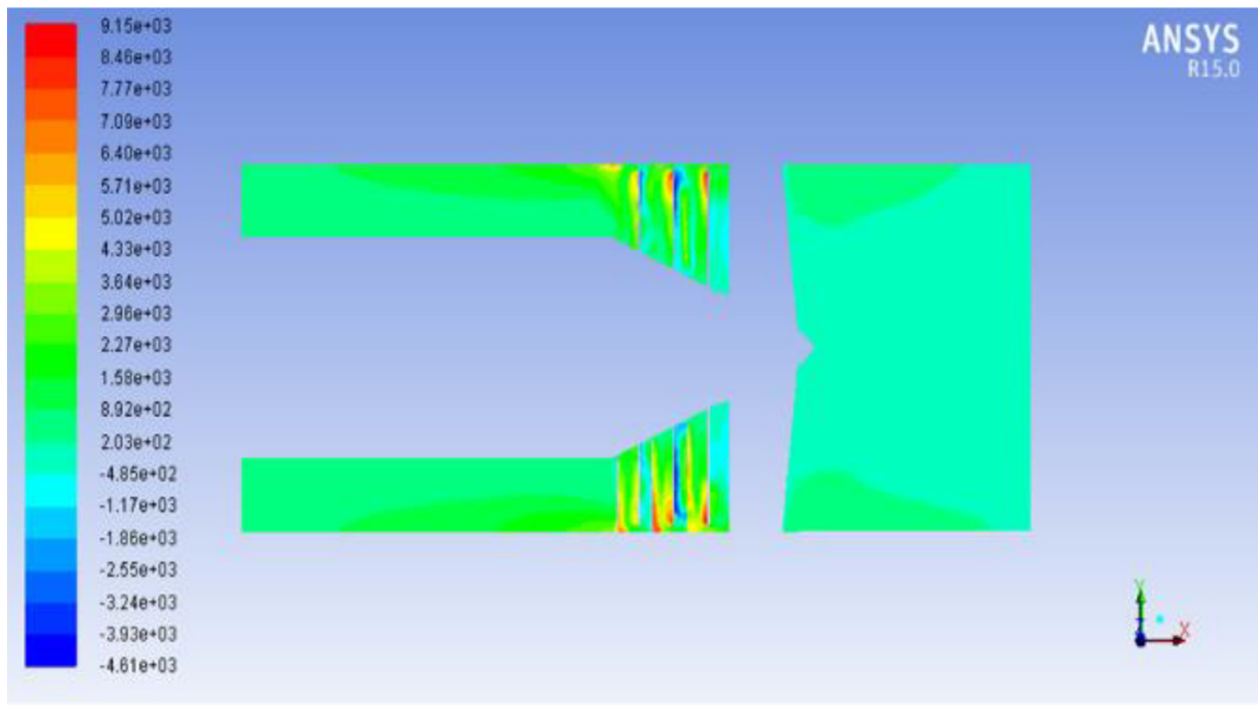

Fig. 8. Contours of total pressure in the optimized model. 


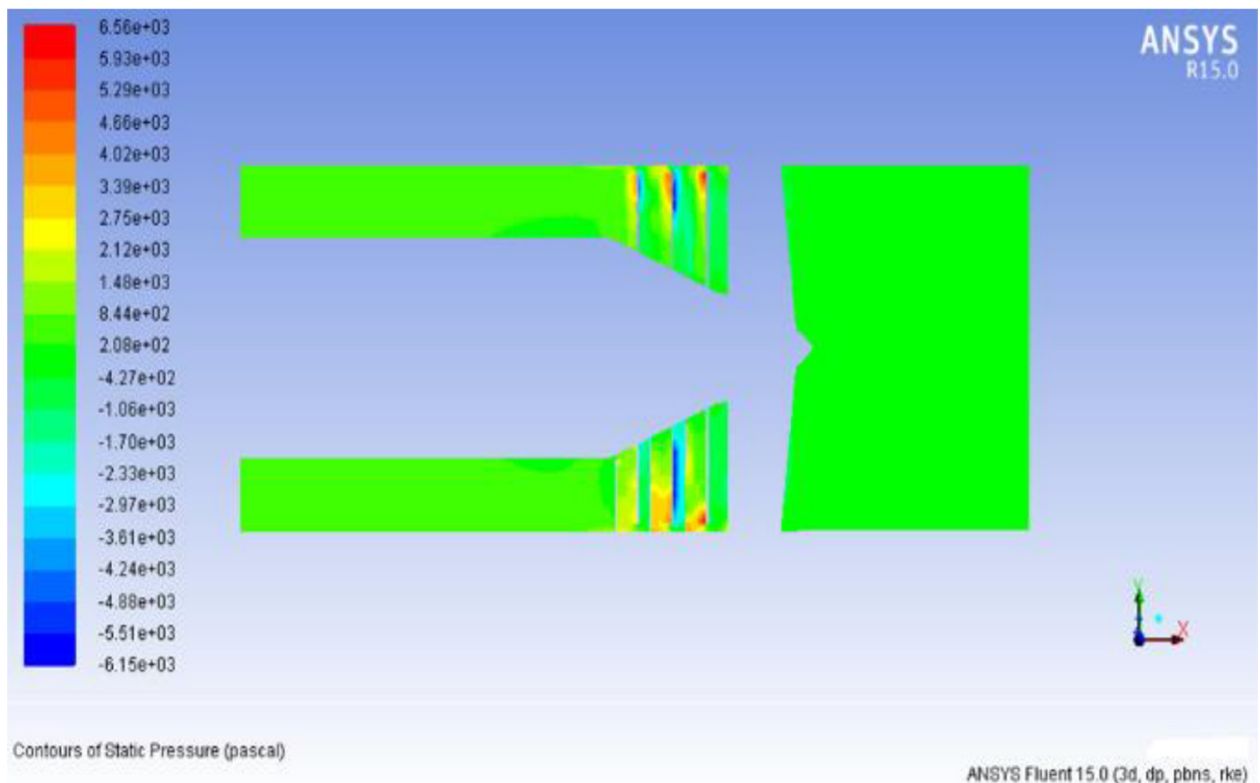

Fig. 9. Contours of static pressure of the optimized model.

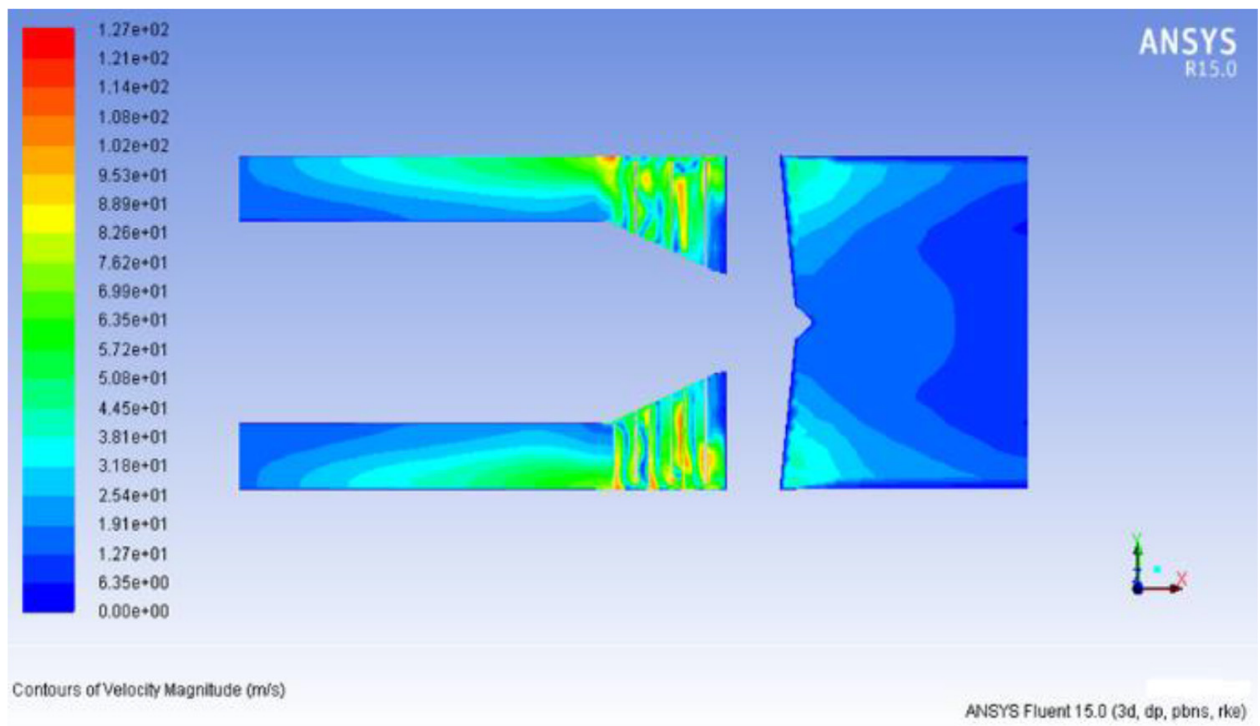

Fig. 10. Velocity magnitude contours for the optimized model.

Due to the counter-rotating compressor design the engine will run in a smooth manner and give desired performance result at various speeds from idle to full power, and engine will run smoothly at various atmospheric conditions. Also specific fuel consumptionwill reduce tremendously due to reduction in weight of an aircraft engine and air fuel burning ratio will increase. Hence, this proposed design can be used in a future gas turbine engine. The main drawback is maintenance and parts availability.

\section{Conclusion}

The counter-rotating compressor rotates with a speed of 7000 RPM consisting of 3 clockwise and 3 anti-clockwise rotating rotors. Static pressure and the velocity of the air is increased by the clockwise rotor. Further, in order to increase the total pressure, the air was circulated to the anti-clockwise rotor wherein the pressure is increased, and the velocity is decreased. The two main designs which we 


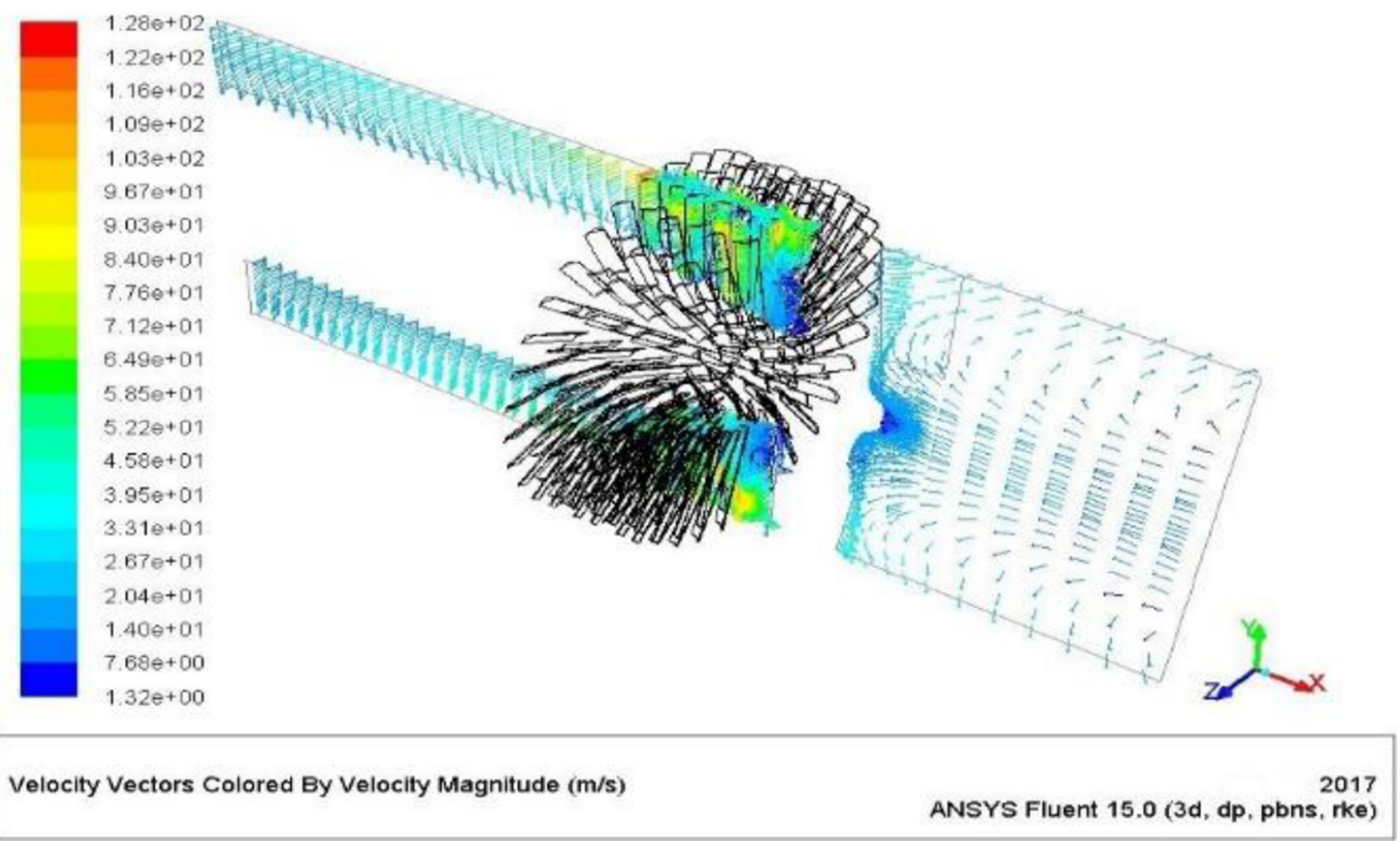

Fig. 11. Velocity vector optimized model.
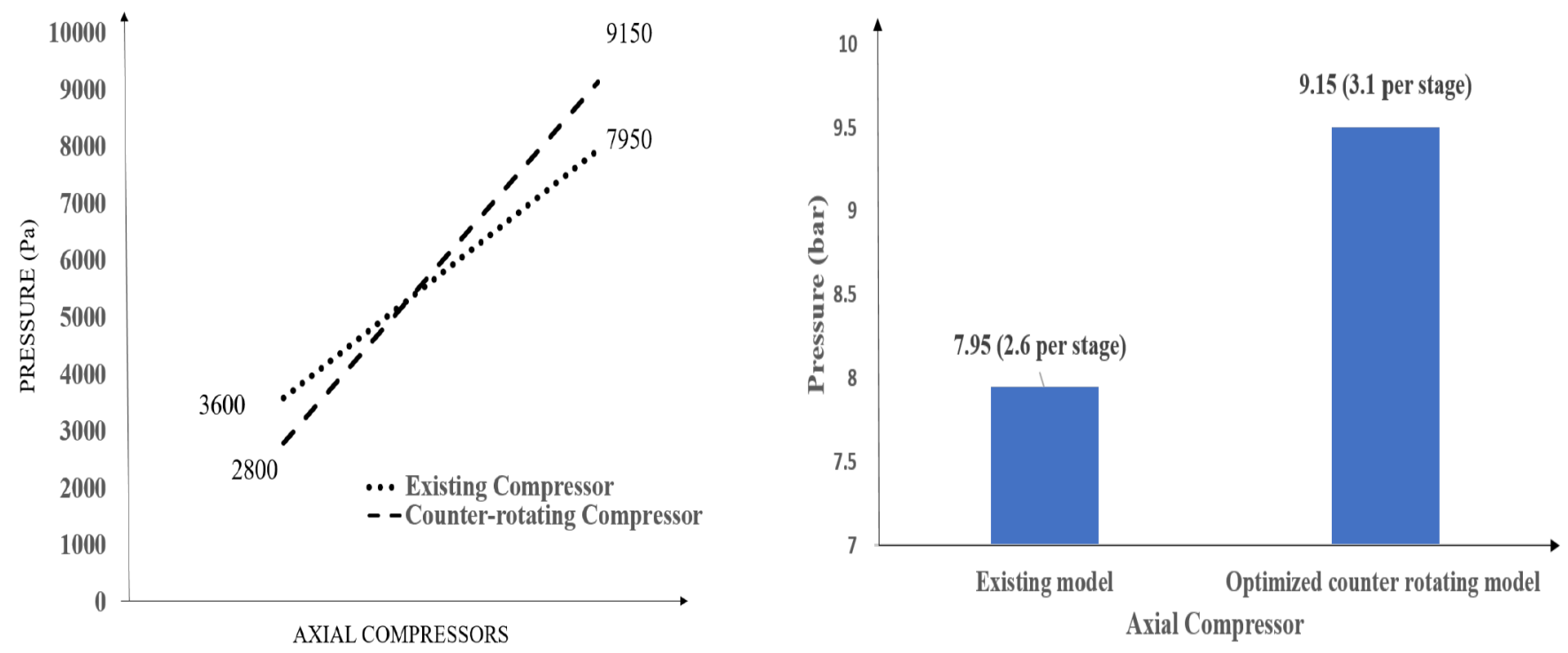

Fig. 12. Represents the comparison result of existing and counter-rotating axial compressor.

have discussed in this paper are that the axial clockwise rotating compressor and the Axial counter-rotating rotor. The regular axial compressor has a pressure ratio of 7.95 and the counter-rotating consists of 9.5. So, it is clear that instead of using a 3-stage axial clockwise compressor, a 2stage counter-rotating compressor can be used in order to achieve the same pressure ratio. This also significantly reduces the weight of the aircraft. Moreover, the other comparison between these two compressors is that the static pressure is more in the counter-rotating $(6500 \mathrm{~Pa})$ than the axial compressor $(3600 \mathrm{~Pa})$. A common factor between these two is that they have high pressure at the front stages of the compressor and another common thing is that the pressure increases at each stage of the rotor. Experimental set up of this counter-rotating compressor will be the future development in this process.

\section{Nomenclature}

r Compressor pressure ratio

Ma Inlet mass flow rate

PV Pressure volume

CFD Computational fluid dynamics

$\eta_{\mathrm{m}} \quad$ Mechanical efficiency

A Area

IGV Inlet guide vanes 
AC Axial compressor

CS Centrifugal compressor

$\rho \quad$ Density

$\mathrm{M}_{\mathrm{f}} \quad$ Mass flow rate of fuel

$\mathrm{Q}_{\mathrm{c}} \quad$ Compressor's exit heat energy

$\mathrm{Q}_{\mathrm{b}} \quad$ Combustion chamber exit heat energy

$\mathrm{C}_{\mathrm{v}} \quad$ Calorific value

$\eta_{\mathrm{t}} \quad$ Efficiency of turbine

$\mathrm{C}_{\mathrm{p}} \quad$ Gas heat capacity at constant pressure

$\mathrm{M}$ Mach number

P Pressure

T Temperature

N Nozzle

CAD Computer aided design

T Turbine

C Compressor

CC Combustion chamber

$\mathrm{T}_{01} \quad$ Compressor inlet temperature

$\mathrm{T}_{02} \quad$ Compressor outlet temperature

$\mathrm{T}_{04} \quad$ Nozzle inlet temperature

$\mathrm{P}_{01} \quad$ Compressor inlet pressure

$\mathrm{P}_{02} \quad$ Compressor outlet pressure

$\mathrm{P}_{03} \quad$ Turbine inlet pressure

$\mathrm{T}_{04} \quad$ Nozzle inlet temperature

$\mathrm{P}_{04} \quad$ Nozzle inlet pressure

F Thrust

\section{References}

1. E. Johann, Rolls Royce Deutschland Ltd and Co KG, Compressor for an aircraft engine. United States patent US $7,207,772,2007$

2. K.H. Lüdtke, Process centrifugal compressors: basics, function, operation, design, application. Springer Science \& Business Media, 2004

3. J.A. Marcos, United Technologies Corp, Control of low compressor vanes and fuel for a gas turbine engine. United States patent US 5,133,182, 1992

4. L.J. Cheshire, The design and development of centrifugal compressors for aircraft gas turbines, Proc. Insti. Mech. Eng. 153, 426-440 (1945)

5. M. Orkisz, S. Stawarz, Modeling of turbine engine axial-flow compressor and turbine characteristics, J. Propuls. Power 16, 336-339 (2000)
6. R. Kervistin, Safran Aircraft Engines SAS. Cooling system for a gas turbine engine compressor. United States patent US $5,297,386,1994$

7. C. Balan, W. Tabakoff, Axial flow compressor performance deterioration, in: 20th Joint Propulsion Conference, 1984, p. 1208

8. S. Kim, C. Son, K. Kim, Combining effect of optimized axial compressor variable guide vanes and bleed air on the thermodynamic performance of aircraft engine system, Energy 119, 199-210 (2017)

9. J.F. Seda, L.W. Dunbar, P.N. Szucs, J.C. Brauer, J.E. Johnson, General Electric Co, Counter rotating aircraft gas turbine engine with high overall pressure ratio compressor. United States patent US 6,732,502, 2004

10. A.H. Stenning, Inlet distortion effects in axial compressors

11. A.F. El-Sayed, Aircraft propulsion and gas turbine engines (CRC Press, 2008)

12. P. Spittle, Gas turbine technology, 2003. http://users.encs. concordia.ca/ kadem/Rolls\%20Royce.pdfS (accessed November 20, 2011)

13. D. Coupe, B.J.G. Dambrine, J.N. Mahieu, Safran aircraft engines SAS, 2015. Aircraft propeller blade, United States Patent $9,162,750$

14. L. Witek, Experimental crack propagation and failure analysis of the first stage compressor blade subjected to vibration, Eng. Fail. Anal. 16, 2163-2170 (2009)

15. A.L. Hutson, M. Niinomi, T. Nicholas, D. Eylon, Effect of various surface conditions on fretting fatigue behavior of Ti-6Al-4V, Int. J. Fatigue 24, 1223-1234 (2002)

16. D. Nowell, D. Dini, D.A. Hills, Recent developments in the understanding of fretting fatigue, Eng. Fract. Mech. 73, 207-222 (2006)

17. N.S. Xi, P.D. Zhong, H.Q. Huang, H. Yan, C.H. Tao, Failure investigation of blade and disk in first stage compressor, Eng. Fail. Anal. 7, 385-392 (2000)

18. A. Kermanpur, H.S. Amin, S. Ziaei-Rad, N. Nourbakhshnia, M. Mosaddeghfar, Failure analysis of TI6AL4V gas turbine compressor blades, Eng. Fail. Anal. 15, 1052-1064 (2008)

19. F. Ferdaus, R. Ganapathi, Computational and experimental testing of aircraft bell nozzle, Int. J. Veh. Struct. Syst. (IJVSS) 11 (2019)

20. F. Ferdaus, R. Sridhar, G. Sathishkumar, S. Sivabalan, Computational analysis of straight nozzle: technical note, Int. J. Veh. Struct. Syst. (2019). DOI: http://dx.doi.org/10.4273/ ijvss.11.2.15

Cite this article as: F. Ferdaus, Nitish Kumar, G. Sakthivel, N. Raghukiran, Stage reduced counter-rotating axial compressor for jet engine, Int. J. Simul. Multidisci. Des. Optim. 12, 3 (2021) 